\title{
Intracerebroventricular Injection of Oxidant and Antioxidant Molecules Affects Long-Term Potentiation in Urethane Anaesthetized Rats
}

\author{
A. Viggiano, E. VIGGiANO, M. MONDA, A. Viggiano, S. ASCIONE, S. AMARO, \\ B. DE LUCA
}

Second University of Naples, Department of Experimental Medicine - Section of Human

Physiology, Naples, Italy

Received February 28, 2006

Accepted February 19, 2007

On-line available April 25, 2007

\begin{abstract}
Summary
Production of superoxide anions in the incubation medium of hippocampal slices can induce long-term potentiation (LTP). Other reactive oxygen species (ROS) such as hydrogen peroxide are able to modulate LTP and are likely to be involved in aging mechanisms. The present study explored whether intracerebroventricular (ICV) injection of oxidant or antioxidant molecules could affect LTP in vivo. With this aim in mind, field excitatory post-synaptic potentials (fEPSPs) elicited by stimulation of the perforant pathway were recorded in the dentate gyrus of the hippocampal formation in urethane-anesthetized rats. $\mathrm{N}$-acetyl-Lcysteine, hydrogen peroxide $\left(\mathrm{H}_{2} \mathrm{O}_{2}\right)$ or hypoxanthine/xanthineoxidase solution (a superoxide producing system) were administrated by ICV injection. The control was represented by a group injected with saline ICV. Ten minutes after the injection, LTP was induced in the granule cells of the dentate gyrus by high frequency stimulation of the perforant pathway. Neither the $\mathrm{H}_{2} \mathrm{O}_{2}$ injection or the $\mathrm{N}$-acetyl-L-cysteine injection caused any variation in the fEPSP at the 10-min post-injection time point, whereas the superoxide generating system caused a significant increase in the fEPSP. Moreover, at $60 \mathrm{~min}$ after tetanic stimulation, all treatments attenuated LTP compared with the control group. These results show that ICV administration of oxidant or antioxidant molecules can modulate LTP in vivo in the dentate gyrus. Particularly, a superoxide producing system can induce potentiation of the synaptic response. Interestingly, ICV injection of oxidants or antioxidants prevented a full expression of LTP compared to the saline injection.
\end{abstract}

\section{Key words}

Oxidant molecules - Antioxidant molecules - Intracerebroventricular injection $\bullet$ Long-term potentiation $\bullet$ Urethane anesthesia

\section{Corresponding author}

B. De Luca, Department of Experimental Medicine, Second University of Naples, Via De Crecchio 8, 80138 Naples, Italy. Fax +39/0815667500. E-mail: bruno.deluca@unina2.it

\section{Introduction}

Reactive oxygen species (ROS) play a normal role as signaling molecules (Suzuki et al. 1997). Growth or pruning of the synaptic spine could be controlled in part by the balance in the synapse between neurodestructive pro-oxidants and neuroprotective antioxidants (Smythies 1999). In hippocampal slices, the induction of long-term potentiation (LTP) in CA1 region (Knapp and Klann 2002b, Kamsler and Segal 2003a) can be modulated by hydrogen peroxide and the increase or decrease of the potentiation is linked to the hydrogen peroxide $\left(\mathrm{H}_{2} \mathrm{O}_{2}\right)$ concentration in the perfusion medium (Pellmar et al. 1991, Auerbach and Segal 1997, Katsuki et al. 1997, Kamsler and Segal 2003a). Moreover, peroxide scavengers (Knapp and Klann 2002a) and superoxide scavengers (Klann et al. 1998, Klann 1998) can prevent full expression of LTP possibly by sequestration of superoxide anions. Notably, superoxide generation by xanthine oxidase activity can induce LTP 
in CA1 region in hippocampal slices (Knapp and Klann 2002a).

In vivo evidence shows that dietary manipulation with antioxidants and stress condition can also influence LTP (McGahon et al. 1999a,b, Vereker et al. 2001, Shakesby et al. 2002, Alfarez et al. 2003). Furthermore, reactive oxygen species (ROS) are possibly related to LTP impairment with aging and this process may be reversed by antioxidant-enriched diet (Murray and Lynch 1998, McGahon et al. 1999a,b, O'Donnell et al. 2000, Viggiano et al. 2006).

To advance in the understanding of the molecular mechanisms underlying LTP, it would be of great interest to support the results obtained from slices with an in vivo whole-brain model. The aim of the present study was to test the effects of ICV injections of oxidant or antioxidant molecules on LTP induced in dentate gyrus (DG) of anesthetized rats.

\section{Methods}

We used male Sprague-Dawley rats weighing 250-300 g and housed at a controlled temperature of $22 \pm 1$ ${ }^{\circ} \mathrm{C}$ and humidity of $70 \%$ with a $12 / 12 \mathrm{~h}$ light-dark cycle from 07:00 to 19:00 h. Laboratory standard food (Mil, Morini, Italy) and water were available ad libitum. All other parameters fulfilled the requirements of the 'Guide for the Care and Use of Laboratory Animals' by the National Research Council, implemented by EU and local rules.

Rats were anesthetized with $1 \mathrm{~g} / \mathrm{kg}$ urethane and placed in a stereotaxic apparatus with lambda $1 \mathrm{~mm}$ below bregma. The body temperature was monitored by a rectal thermometer and maintained at $37.0 \pm 0.2{ }^{\circ} \mathrm{C}$ by an electrically shielded heating pad. In order to record evoked potentials in the hilus of DG, two small holes were drilled into the bone to reach the perforant pathway (pp) $(7.5 \mathrm{~mm}$ posterior to the bregma, $4.2 \mathrm{~mm}$ lateral to the midline) with an unipolar stimulating electrode and the DG $(3.5 \mathrm{~mm}$ posterior to the bregma, $2.5 \mathrm{~mm}$ lateral to the midline) with the recording electrode (Paxinos and Watson 1997). Two screws in the occipital bone were used as reference and ground. A third hole was made to insert a cannula into the lateral ventricle $(0.4 \mathrm{~mm}$ posterior to the bregma, $1.7 \mathrm{~mm}$ lateral to the midline, $3.3 \mathrm{~mm}$ from the cranial theca). Recording and stimulating electrodes were always on the same side of the brain as the cannula.

Stimulation and recording was achieved with a modular analog instrumentation (Neurolog, Digitimer,

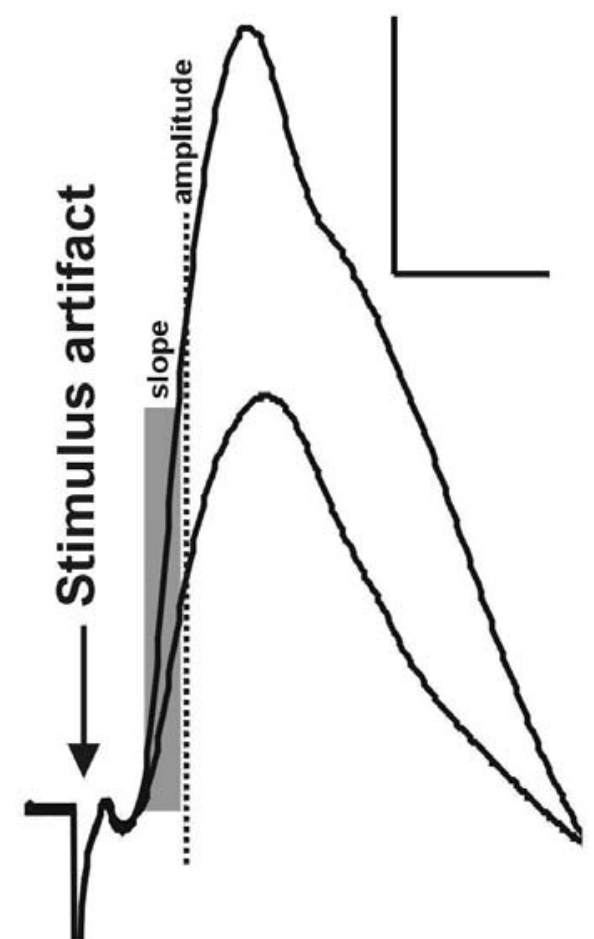

Fig. 1. Example of fEPSP before and after LTP induction. Arrow marks the stimulus artifact, grey square marks the portion of fEPSP used in evaluating slope (1 ms). At fixed latency $(3.0 \mathrm{~ms})$ from the start of stimulus artifact, amplitude of fEPSP was calculated. Calibration bars: $2 \mathrm{mV} ; 4 \mathrm{~ms}$.

England) connected to a $\mathrm{PC}$ with a $\mathrm{A} / \mathrm{D}-\mathrm{D} / \mathrm{A}$ converter (AT-MIO-XE50, National Instruments, TX, USA) to automatically follow the whole stimulating/recording protocol with a custom software written under LabView environment (National Instruments).

The depth of recording and stimulating electrodes was optimized to maximize the amplitude of fEPSP evoked by a perforant path test shock.

For eliciting fEPSP, a series of eight monopolar pulses was generated with a frequency of $0.1 \mathrm{~Hz}$. The pulse width was $100 \mu$ s and the stimulus intensity (200-400 $\mu \mathrm{A})$ was adjusted to the value that evoked $50 \%$ of the maximum fEPSP amplitude. Amplitude (measured at 3.0 ms from the stimulus artifact; maximal amplitude never occurred before $3.8 \mathrm{~ms}$ ) and slope (on the first millisecond of the rising phase) of the fEPSP were used to quantify the evoked potentials.

LTP was induced by stimulating the pp with 20 trains of 15 impulses with the same weight and amplitude as the test pulse. The frequency within the train was 200 $\mathrm{Hz}$, and the distance between the trains was $5 \mathrm{~s}$ (Krug et al. 2001). Examples of fEPSP before and after LTP with measured parameters are shown in Figure 1.

Four groups of five animals each were subjected 

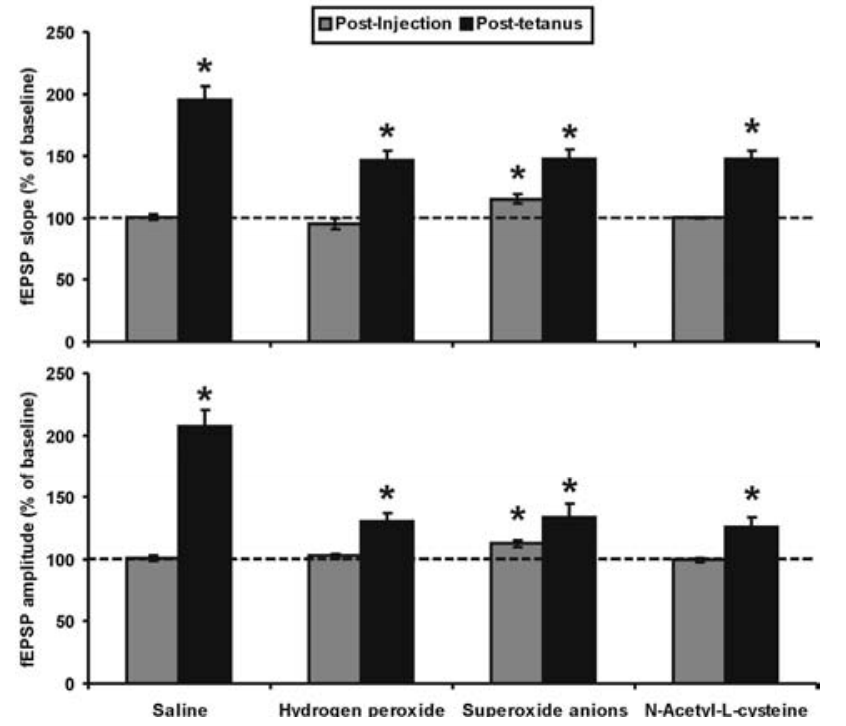

Fig. 2. Percent of variation in fEPSP after $10 \mathrm{~min}$ from ICV injection of $5 \mu \mathrm{l}$ of saline, hydrogen peroxide $(8.8 \mathrm{mM})$, hypoxanthine $(1 \mathrm{mM}) /$ xanthine oxidase $(0.82 \mathrm{U} / \mathrm{ml}$ ) (superoxide producing system) or N-Acetyl-L-cysteine $(100 \mathrm{mM})$ and after 60 min after high frequency stimulation in the same groups. The values are means \pm S.E.M. of five observations in each case. Asterics mark significant differences with respect to saline group.

to the following procedure: 30 min recovery, 8 -fEPSP $(0.1$ $\mathrm{Hz}$ ) sampling, $5 \mu \mathrm{l}$ intracerebroventricular (ICV) injection of one of the solutions described below, 10 min elapsed time, 8-fEPSP $(0.1 \mathrm{~Hz})$ sampling, LTP induction, 8-fEPSP $(0.1 \mathrm{~Hz})$ sampling after $60 \mathrm{~min}$ from the high frequency stimulation.

The solutions were as follows: saline (group 1), N-acetyl-L-cysteine (NAC) (100 mM) (group 2), hydrogen peroxide $(8.8 \mathrm{mM})$ (group 3), and hypoxanthine (1 $\mathrm{mM}$ )/xanthine-oxidase $(0.82 \mathrm{U} / \mathrm{ml})$ solution (superoxide anions generating system) (group 4). The solutions were always prepared immediately before injection.

At the end of the experimental session, animals were overdosed with urethane and perfused through the heart with $0.9 \%$ saline followed by $10 \%$ formalin in PBS. All electrode sites were histologically verified in $50 \mu \mathrm{m}$ frozen sections stained with cresyl violet.

One-way analysis of variance (ANOVA) was used to test significant differences between the experimental conditions. Paired $t$ test was used to compare the pre-treatment state with post-treatment state. Rejection level was fixed at $\mathrm{p}=0.05$.

\section{Results}

Figure 2 shows the percentage variation in fEPSP after 10 min from injection and after $60 \mathrm{~min}$ after high frequency stimulation and displays the summary of the fEPSP data.

The injection of hypoxanthine/xanthine-oxidase caused a significant increase of the fEPSP amplitude $(112.8 \pm 2.8)$ and slope $(115.4 \pm 3.8) \quad[P<0.01$; paired $t$ test]. On the other hand, ICV injection of saline, hydrogen peroxide $(8.8 \mathrm{mM})$ or NAC did not affect the fEPSP amplitude and slope.

Sixty minutes after high frequency stimulation, significant differences appeared in fEPSP amplitude $[\mathrm{F}(3,16)=14.69 ; P<0.01$ one way ANOVA $]$ and in fEPSP slope $[\mathrm{F}(3,16)=7.87 ; \quad P<0.01 ;$ one way ANOVA]. Unplanned pair-wise comparisons with Tukey's method revealed that the potentiation in all the experimental groups was significantly lower than in the control group [saline group (amplitude 207.0 \pm 13.8 ; slope 195.1 \pm 11.4$)$ ], but there was no significant difference among the three experimental conditions $\left[\mathrm{H}_{2} \mathrm{O}_{2}\right.$ group (amplitude 131.0 \pm 5.9 ; slope 146.7 \pm 7.6 ); the superoxide group (133.9 $\pm 11.0 ; 148.3 \pm 7.0)$; NAC group (125.8 \pm 7.7 ;

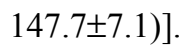

\section{Discussion}

These results show that the ICV injection of oxidant or antioxidant molecules can modulate the synaptic transmission in DG in vivo. The hypoxanthine/xanthine-oxidase system, a system able to produce superoxide anions (Frederiks and Bosch 1997), could induce potentiation in DG while hydrogen peroxide and the antioxidant NAC attenuated LTP. Similar results were obtained in vivo by Vereker and coworkers (O'Donnell et al. 2000, Vereker et al. 2001) who showed that hydrogen peroxide inhibits LTP induction. These authors also suggested protective effects of antioxidants on aging and stress impairment in LTP, because the addition of antioxidants to the standard laboratory diet reverses the age-related deficit in LTP (McGahon et al. 1999a,b, Vereker et al. 2001, Martin et al. 2002). Our results show that ICV injection of an antioxidant species (NAC) has a deleterious effect on LTP. This result agrees with the evidence that antioxidants have inhibitory effects on LTP in vitro (Klann et al. 1998, Klann 1998, Thiels et al. 2000) and that the superoxide production is needed for LTP in CA1 in hippocampal slices (Knapp and Klann 2002a). Moreover, our results show that even if the hypoxanthine/xanthine-oxidase system was able to induce potentiation, its effect on LTP induced by tetanic stimulation was deleterious because the overall 
potentiation after 60 min from tetanus was significantly smaller than in the control. It seems reasonable that superoxide produced by exogenous xanthine-oxidase activity leads to production of hydrogen peroxide with deleterious effects for LTP (Knapp and Klann 2002b), but this hypothesis needs further studies. The inhibitory effect of hydrogen peroxide on LTP is well known and Kamsler and Segal (2003a,b) proposed a dual role for $\mathrm{H}_{2} \mathrm{O}_{2}$ with high concentrations suppressing LTP and low concentration enhancing it. In our model, the $\mathrm{H}_{2} \mathrm{O}_{2}$ injection did not affect the fEPSP slope and amplitude as in the in vitro slice preparations, but had a considerable negative effect on the LTP induction by tetanic stimulation. These differences between the in vivo model and in vitro slices preparation are not explainable with the present data and also need further evaluations. Nonetheless, they stress the importance of validating and corroborating in vitro data with in vivo models. Some observations should be performed with regard to the ICV injection and anesthesia. It was not possible to determine the distribution of the injected substances through the brain parenchyma so that it was not possible to evaluate the effective concentration of the oxidants or antioxidants at the level of DG in the present model. It is also known that under urethane anesthesia the induction of LTP requires a stronger tetanization protocol than in freely moving rats (Riedel et al. 1994). Therefore it could be interesting to evaluate the effects of oxidants and antioxidants in freely moving rats.
Two limitations of the present study should be pointed out. The first one concerns the absence of a doseresponse curve that will be obtained by future experiments. In fact, it seems important to investigate the effect of different ICV oxidants and antioxidants doses on LTP considering that $\mathrm{H}_{2} \mathrm{O}_{2}$ modulation of LTP depends on the concentration (Kamsler and Segal 2003a,b). The second limit is related to the lack of a complete time course of the potentiation. This protocol was followed by the authors to simplify the design of the experimental session. Again, further studies are planned to obtain a complete time course for the effects of ROS ICV injection on LTP.

The principal finding of this study is that the intracerebroventricular injection of oxidant and antioxidant molecules is able to modulate long-term potentiation in dentate gyrus during acute recording in vivo. With the discussed limits, this finding supports and extends the validity of previous data obtained from in vitro slice preparations.

\section{Conflict of Interest}

There is no conflict of interest.

\section{Acknowledgements}

The authors would like to thank Dr. Jan Bures for critical reading of the manuscript. They also thank the anonymous referees for their constructive comments.

\section{References}

ALFAREZ DN, JOELS M, KRUGERS HJ: Chronic unpredictable stress impairs long-term potentiation in rat hippocampal CA1 area and dentate gyrus in vitro. Eur J Neurosci 17: 1928-1934, 2003.

AUERBACH JM, SEGAL M: Peroxide modulation of slow onset potentiation in rat hippocampus. J Neurosci 17: 8695-8701, 1997.

FREDERIKS WM, BOSCH KS: Localization of superoxide dismutase activity in rat tissues. Free Radic Biol Med 22: 241-248, 1997.

KAMSLER A, SEGAL M: Hydrogen peroxide modulation of synaptic plasticity. J Neurosci 23: 269-276, 2003 a.

KAMSLER A, SEGAL M: Paradoxical actions of hydrogen peroxide on long-term potentiation in transgenic superoxide dismutase-1 mice. $J$ Neurosci 23: 10359-10367, 2003 b.

KATSUKI H, NAKANISHI C, SAITO H, MATSUKI N: Biphasic effect of hydrogen peroxide on field potentials in rat hippocampal slices. Eur J Pharmacol 337: 213-218, 1997.

KLANN E: Cell-permeable scavengers of superoxide prevent long-term potentiation in hippocampal area CA1. J Neurophysiol 80: 452-457, 1998.

KLANN E, ROBERSON ED, KNAPP LT, SWEATT JD: A role for superoxide in protein kinase C activation and induction of long-term potentiation. J Biol Chem 273: 4516-4522, 1998.

KNAPP LT, KLANN E: Potentiation of hippocampal synaptic transmission by superoxide requires the oxidative activation of protein kinase C. J Neurosci 22: 674-683, 2002a. 
KNAPP LT, KLANN E: Role of reactive oxygen species in hippocampal long-term potentiation: contributory or inhibitory? J Neurosci Res 70: 1-7, 2002b.

KRUG M, BRODEMANN R, MATTHIES R, RUTHRICH H, WAGNER M: Activation of the dentate gyrus by stimulation of the contralateral perforant pathway: evoked potentials and long-term potentiation after ipsi- and contralateral induction. Hippocampus 11: 157-167, 2001.

MARTIN DS, SPENCER P, HORROBIN DF, LYNCH MA: Long-term potentiation in aged rats is restored when the age-related decrease in polyunsaturated fatty acid concentration is reversed. Prostaglandins Leukot Essent Fatty Acids 67: 121-130, 2002.

MCGAHON BM, MARTIN DS, HORROBIN DF, LYNCH MA: Age-related changes in LTP and antioxidant defenses are reversed by an alpha-lipoic acid-enriched diet. Neurobiol Aging 20: 655-664, 1999a.

MCGAHON BM, MURRAY CA, HORROBIN DF, LYNCH MA: Age-related changes in oxidative mechanisms and LTP are reversed by dietary manipulation. Neurobiol Aging 20: 643-653, 1999b.

MURRAY CA, LYNCH MA: Dietary supplementation with vitamin E reverses the age-related deficit in long term potentiation in dentate gyrus. J Biol Chem 273: 12161-12168, 1998.

O'DONNELL E, VEREKER E, LYNCH MA: Age-related impairment in LTP is accompanied by enhanced activity of stress-activated protein kinases: analysis of underlying mechanisms. Eur J Neurosci 12: 345-352, 2000.

PAXINOS G, WATSON C: The Rat Brain in Stereotaxic Coordinates, Academic Press, San Diego, 1997.

PELLMAR TC, HOLLINDEN GE, SARVEY JM: Free radicals accelerate the decay of long-term potentiation in field CA1 of guinea-pig hippocampus. Neuroscience 44: 353-359, 1991.

RIEDEL G, SEIDENBECHER T, REYMANN KG: LTP in hippocampal CA1 of urethane-narcotized rats requires stronger tetanization parameters. Physiol Behav 55: 1141-1146, 1994.

SHAKESBY AC, ANWYL R, ROWAN MJ: Overcoming the effects of stress on synaptic plasticity in the intact hippocampus: rapid actions of serotonergic and antidepressant agents. $J$ Neurosci 22: 3638-3644, 2002.

SMYTHIES J: Redox mechanisms at the glutamate synapse and their significance: a review. Eur J Pharmacol 370: 1-7, 1999.

SUZUKI YJ, FORMAN HJ, SEVANIAN A: Oxidants as stimulators of signal transduction. Free Radic Biol Med 22: 269-285, 1997.

THIELS E, URBAN NN, GONZALEZ-BURGOS GR, KANTEREWICZ BI, BARRIONUEVO G, CHU CT, OURY TD, KLANN E: Impairment of long-term potentiation and associative memory in mice that overexpress extracellular superoxide dismutase. $J$ Neurosci 20: 7631-7639, 2000.

VEREKER E, O'DONNELL E, LYNCH A, KELLY A, NOLAN Y, LYNCH MA: Evidence that interleukin-1beta and reactive oxygen species production play a pivotal role in stress-induced impairment of LTP in the rat dentate gyrus. Eur J Neurosci 14: 1809-1819, 2001.

VIGGIANO A, VIGGIANO A, MONDA M, TURCO I, INCARNATO L, VINNO V, VIGGIANO E, BACCARI ME, DE LUCA B: Annurca apple-rich diet restores long-term potentiation and induces behavioral modifications in aged rats. Exp Neurol 199: 354-361, 2006. 